\title{
JENSEN'S TYPE TRACE INEQUALITIES FOR CONVEX FUNCTIONS OF SELFADJOINT OPERATORS IN HILBERT SPACES *
}

\author{
Sever Silvestru Dragomir
}

\begin{abstract}
Some Jensen's type trace inequalities for convex functions of selfadjoint operators in Hilbert spaces are provided. Applications for some convex functions of interest are also given.
\end{abstract}

Keywords: Trace class operators; Hilbert-Schmidt operators; Trace; Convex functions; Jensen's inequality; Trace inequalities for matrices

\section{Introduction}

Let $(H,\langle\cdot, \cdot\rangle)$ be a complex Hilbert space and $\left\{e_{i}\right\}_{i \in I}$ an orthonormal basis of $H$. We say that $A \in \mathcal{B}(H)$ is a Hilbert-Schmidt operator if

$$
\sum_{i \in I}\left\|A e_{i}\right\|^{2}<\infty
$$

It is well know that, if $\left\{e_{i}\right\}_{i \in I}$ and $\left\{f_{j}\right\}_{j \in J}$ are orthonormal bases for $H$ and $A \in$ $\mathcal{B}(H)$ then

$$
\sum_{i \in I}\left\|A e_{i}\right\|^{2}=\sum_{j \in I}\left\|A f_{j}\right\|^{2}=\sum_{j \in I}\left\|A^{*} f_{j}\right\|^{2}
$$

showing that the definition (1.1) is independent of the orthonormal basis and $A$ is a Hilbert-Schmidt operator iff $A^{*}$ is a Hilbert-Schmidt operator.

Received May 11, 2016; accepted September 18, 2016

2010 Mathematics Subject Classification. Primary 47A63; Secondary 47A99.

* The author was supported in part by DST-NRF Centre of Excellence in the Mathematical and Statistical Sciences, School of Computer Science and Applied Mathematics, University of the Witwatersrand, Johannesburg, Private Bag 3, Wits 2050, 
Let $\mathcal{B}_{2}(H)$ the set of Hilbert-Schmidt operators in $\mathcal{B}(H)$. For $A \in \mathcal{B}_{2}(H)$ we define

$$
\|A\|_{2}:=\left(\sum_{i \in I}\left\|A e_{i}\right\|^{2}\right)^{1 / 2}
$$

for $\left\{e_{i}\right\}_{i \in I}$ an orthonormal basis of $H$. This definition does not depend on the choice of the orthonormal basis.

Using the triangle inequality in $l^{2}(I)$, one checks that $\mathcal{B}_{2}(H)$ is a vector space and that $\|\cdot\|_{2}$ is a norm on $\mathcal{B}_{2}(H)$, which is usually called in the literature as the Hilbert-Schmidt norm.

Denote the modulus of an operator $A \in \mathcal{B}(H)$ by $|A|:=\left(A^{*} A\right)^{1 / 2}$.

Because $\||A| x\|=\|A x\|$ for all $x \in H, A$ is Hilbert-Schmidt iff $|A|$ is HilbertSchmidt and $\|A\|_{2}=\||A|\|_{2}$. From (1.2) we have that if $A \in \mathcal{B}_{2}(H)$, then $A^{*} \in$ $\mathcal{B}_{2}(H)$ and $\|A\|_{2}=\left\|A^{*}\right\|_{2}$.

The following theorem collects some of the most important properties of HilbertSchmidt operators:

Theorem 1.1. We have

(i) $\left(\mathcal{B}_{2}(H),\|\cdot\|_{2}\right)$ is a Hilbert space with inner product

$$
\langle A, B\rangle_{2}:=\sum_{i \in I}\left\langle A e_{i}, B e_{i}\right\rangle=\sum_{i \in I}\left\langle B^{*} A e_{i}, e_{i}\right\rangle
$$

and the definition does not depend on the choice of the orthonormal basis $\left\{e_{i}\right\}_{i \in I}$;

(ii) We have the inequalities

$$
\|A\| \leq\|A\|_{2}
$$

for any $A \in \mathcal{B}_{2}(H)$ and

$$
\|A T\|_{2},\|T A\|_{2} \leq\|T\|\|A\|_{2}
$$

for any $A \in \mathcal{B}_{2}(H)$ and $T \in \mathcal{B}(H)$;

(iii) $\mathcal{B}_{2}(H)$ is an operator ideal in $\mathcal{B}(H)$, i.e.

$$
\mathcal{B}(H) \mathcal{B}_{2}(H) \mathcal{B}(H) \subseteq \mathcal{B}_{2}(H) ;
$$

(iv) $\mathcal{B}_{\text {fin }}(H)$, the space of operators of finite rank, is a dense subspace of $\mathcal{B}_{2}(H)$; $H$.

(v) $\mathcal{B}_{2}(H) \subseteq \mathcal{K}(H)$, where $\mathcal{K}(H)$ denotes the algebra of compact operators on

If $\left\{e_{i}\right\}_{i \in I}$ an orthonormal basis of $H$, we say that $A \in \mathcal{B}(H)$ is trace class if

$$
\|A\|_{1}:=\sum_{i \in I}\left\langle|A| e_{i}, e_{i}\right\rangle<\infty
$$


The definition of $\|A\|_{1}$ does not depend on the choice of the orthonormal basis $\left\{e_{i}\right\}_{i \in I}$. We denote by $\mathcal{B}_{1}(H)$ the set of trace class operators in $\mathcal{B}(H)$.

The following proposition holds:

Proposition 1.1. If $A \in \mathcal{B}(H)$, then the following are equivalent:

(i) $A \in \mathcal{B}_{1}(H)$;

(ii) $|A|^{1 / 2} \in \mathcal{B}_{2}(H)$;

(ii) $A($ or $|A|)$ is the product of two elements of $\mathcal{B}_{2}(H)$.

The following properties are also well known:

Theorem 1.2. With the above notations:

(i) We have

$$
\|A\|_{1}=\left\|A^{*}\right\|_{1} \text { and }\|A\|_{2} \leq\|A\|_{1}
$$

for any $A \in \mathcal{B}_{1}(H)$;

(ii) $\mathcal{B}_{1}(H)$ is an operator ideal in $\mathcal{B}(H)$, i.e.

$$
\mathcal{B}(H) \mathcal{B}_{1}(H) \mathcal{B}(H) \subseteq \mathcal{B}_{1}(H) ;
$$

(iii) We have

$$
\mathcal{B}_{2}(H) \mathcal{B}_{2}(H)=\mathcal{B}_{1}(H)
$$

(iv) We have

$$
\|A\|_{1}=\sup \left\{\langle A, B\rangle_{2} \mid B \in \mathcal{B}_{2}(H),\|B\| \leq 1\right\} ;
$$

(v) $\left(\mathcal{B}_{1}(H),\|\cdot\|_{1}\right)$ is a Banach space.

(iv) We have the following isometric isomorphisms

$$
\mathcal{B}_{1}(H) \cong K(H)^{*} \text { and } \mathcal{B}_{1}(H)^{*} \cong \mathcal{B}(H),
$$

where $K(H)^{*}$ is the dual space of $K(H)$ and $\mathcal{B}_{1}(H)^{*}$ is the dual space of $\mathcal{B}_{1}(H)$.

We define the trace of a trace class operator $A \in \mathcal{B}_{1}(H)$ to be

$$
\operatorname{tr}(A):=\sum_{i \in I}\left\langle A e_{i}, e_{i}\right\rangle,
$$

where $\left\{e_{i}\right\}_{i \in I}$ an orthonormal basis of $H$. Note that this coincides with the usual definition of the trace if $H$ is finite-dimensional. We observe that the series (1.9) converges absolutely and it is independent from the choice of basis.

The following result collects some properties of the trace: 
Theorem 1.3. We have

(i) If $A \in \mathcal{B}_{1}(H)$ then $A^{*} \in \mathcal{B}_{1}(H)$ and

$$
\operatorname{tr}\left(A^{*}\right)=\overline{\operatorname{tr}(A)}
$$

(ii) If $A \in \mathcal{B}_{1}(H)$ and $T \in \mathcal{B}(H)$, then $A T, T A \in \mathcal{B}_{1}(H)$ and

$$
\operatorname{tr}(A T)=\operatorname{tr}(T A) \text { and }|\operatorname{tr}(A T)| \leq\|A\|_{1}\|T\| ;
$$

(iii) $\operatorname{tr}(\cdot)$ is a bounded linear functional on $\mathcal{B}_{1}(H)$ with $\|\operatorname{tr}\|=1$;

(iv) If $A, B \in \mathcal{B}_{2}(H)$ then $A B, B A \in \mathcal{B}_{1}(H)$ and $\operatorname{tr}(A B)=\operatorname{tr}(B A)$;

(v) $\mathcal{B}_{\text {fin }}(H)$ is a dense subspace of $\mathcal{B}_{1}(H)$.

Utilising the trace notation we obviously have that

$$
\langle A, B\rangle_{2}=\operatorname{tr}\left(B^{*} A\right)=\operatorname{tr}\left(A B^{*}\right) \text { and }\|A\|_{2}^{2}=\operatorname{tr}\left(A^{*} A\right)=\operatorname{tr}\left(|A|^{2}\right)
$$

for any $A, B \in \mathcal{B}_{2}(H)$.

For the theory of trace functionals and their applications the reader is referred to $[37]$.

For some classical trace inequalities see [5], [7], [34] and [48], which are continuations of the work of Bellman [2]. For related works the reader can refer to [1], [3], [5], [28], [31], [32], [33], [35] and [45].

Consider the orthonormal basis $\mathcal{E}:=\left\{e_{i}\right\}_{i \in I}$ in the complex Hilbert space $(H,\langle\cdot, \cdot\rangle)$ and for a nonzero operator $B \in \mathcal{B}_{2}(H)$ let introduce the subset of indices from $I$ defined by

$$
I_{\mathcal{E}, B}:=\left\{i \in I: B e_{i} \neq 0\right\} .
$$

We observe that $I_{\mathcal{E}, B}$ is non-empty for any nonzero operator $B$ and if $\operatorname{ker}(B)=0$, i.e. $B$ is injective, then $I_{\mathcal{E}, B}=I$. We also have for $B \in \mathcal{B}_{2}(H)$ that

$$
\operatorname{tr}\left(|B|^{2}\right)=\operatorname{tr}\left(B^{*} B\right)=\sum_{i \in I}\left\langle B^{*} B e_{i}, e_{i}\right\rangle=\sum_{i \in I}\left\|B e_{i}\right\|^{2}=\sum_{i \in I_{\mathcal{E}, B}}\left\|B e_{i}\right\|^{2} .
$$

In the recent paper [26] we obtained, among others, the following result for convex functions:

Theorem 1.4. Let $A$ be a selfadjoint operator on the Hilbert space $H$ and assume that $\operatorname{Sp}(A) \subseteq[m, M]$ for some scalars $m, M$ with $m<M$. If $f$ is a continuous convex function on $[m, M], \mathcal{E}:=\left\{e_{i}\right\}_{i \in I}$ is an orthonormal basis in $H$ and $B \in$ $\mathcal{B}_{2}(H) \backslash\{0\}$, then $\frac{\operatorname{tr}\left(|B|^{2} A\right)}{\operatorname{tr}\left(|B|^{2}\right)} \in[m, M]$ and

$$
\begin{gathered}
f\left(\frac{\operatorname{tr}\left(|B|^{2} A\right)}{\operatorname{tr}\left(|B|^{2}\right)}\right) \operatorname{tr}\left(|B|^{2}\right) \\
\leq J_{\mathcal{E}}(f ; A, B) \leq \operatorname{tr}\left(|B|^{2} f(A)\right) \\
\leq \frac{1}{M-m}\left(f(m) \operatorname{tr}\left[|B|^{2}\left(M 1_{H}-A\right)\right]+f(M) \operatorname{tr}\left[|B|^{2}\left(A-m 1_{H}\right)\right]\right),
\end{gathered}
$$


where

$$
J_{\mathcal{E}}(f ; A, B):=\sum_{i \in I_{\mathcal{E}, B}} f\left(\frac{\left\langle B^{*} A B e_{i}, e_{i}\right\rangle}{\left\|B e_{i}\right\|^{2}}\right)\left\|B e_{i}\right\|^{2} .
$$

For related functionals and their superadditivity and monotonicity properties see $[26]$.

For some inequalities for convex functions see [8]-[12], [27] and [44]. For inequalities for functions of selfadjoint operators, see [14]-[23], [38], [40], [41], [42], [43] and the books [24], [25] and [29].

Motivated by the above results we establish in this paper other trace inequalities for convex functions of selfadjoint operators. Some examples for convex functions of interest are also given.

\section{New Inequalities for Convex Functions}

We recall the gradient inequality for the convex function $f:[m, M] \rightarrow \mathbb{R}$, namely

$$
f(\varsigma)-f(\tau) \geq \delta_{f}(\tau)(\varsigma-\tau)
$$

for any $\varsigma, \tau \in[m, M]$ where $\delta_{f}(\tau) \in\left[f_{-}^{\prime}(\tau), f_{+}^{\prime}(\tau)\right]$, (for $\tau=m$ we take $\delta_{f}(\tau)=$ $f_{+}^{\prime}(m)$ and for $\tau=M$ we take $\left.\delta_{f}(\tau)=f_{-}^{\prime}(M)\right)$. Here $f_{+}^{\prime}(m)$ and $f_{-}^{\prime}(M)$ are the lateral derivatives of the convex function $f$.

The following result holds:

Theorem 2.1. Let $A$ be a selfadjoint operator on the Hilbert space $H$ and assume that $\operatorname{Sp}(A) \subseteq[m, M]$ for some scalars $m, M$ with $m<M$. If $f$ is a continuous convex function on $[m, M]$ and $B \in \mathcal{B}_{2}(H) \backslash\{0\}$, then we have $\frac{\operatorname{tr}\left(|B|^{2} A\right)}{\operatorname{tr}\left(|B|^{2}\right)} \in[m, M]$,

$$
\begin{aligned}
& \delta_{f}\left(\frac{\operatorname{tr}\left(|B|^{2} A\right)}{\operatorname{tr}\left(|B|^{2}\right)}\right) \frac{\operatorname{tr}\left(\left|B^{*}\right|^{2} A\right)-\operatorname{tr}\left(|B|^{2} A\right)}{\operatorname{tr}\left(|B|^{2}\right)} \\
& \leq \frac{\operatorname{tr}\left(\left|B^{*}\right|^{2} f(A)\right)}{\operatorname{tr}\left(|B|^{2}\right)}-f\left(\frac{\operatorname{tr}\left(|B|^{2} A\right)}{\operatorname{tr}\left(|B|^{2}\right)}\right)
\end{aligned}
$$

where

$$
\delta_{f}\left(\frac{\operatorname{tr}\left(|B|^{2} A\right)}{\operatorname{tr}\left(|B|^{2}\right)}\right) \in\left[f_{-}^{\prime}\left(\frac{\operatorname{tr}\left(|B|^{2} A\right)}{\operatorname{tr}\left(|B|^{2}\right)}\right), f_{+}^{\prime}\left(\frac{\operatorname{tr}\left(|B|^{2} A\right)}{\operatorname{tr}\left(|B|^{2}\right)}\right)\right]
$$

and the Jensen's inequality

$$
f\left(\frac{\operatorname{tr}\left(|B|^{2} A\right)}{\operatorname{tr}\left(|B|^{2}\right)}\right) \leq \frac{\operatorname{tr}\left(|B|^{2} f(A)\right)}{\operatorname{tr}\left(|B|^{2}\right)} .
$$


Proof. Let $\mathcal{E}:=\left\{e_{i}\right\}_{i \in I}$ be an orthonormal basis in $H$.

Utilising the gradient inequality (2.1) we get

$$
f(\varsigma)-f\left(\frac{\operatorname{tr}\left(|B|^{2} A\right)}{\operatorname{tr}\left(|B|^{2}\right)}\right) \geq \delta_{f}\left(\frac{\operatorname{tr}\left(|B|^{2} A\right)}{\operatorname{tr}\left(|B|^{2}\right)}\right)\left(\varsigma-\frac{\operatorname{tr}\left(|B|^{2} A\right)}{\operatorname{tr}\left(|B|^{2}\right)}\right)
$$

for any $\varsigma \in[m, M]$, since obviously, by $S p(A) \subseteq[m, M]$ we have

$$
m\left\|B e_{i}\right\|^{2} \leq\left\langle A B e_{i}, B e_{i}\right\rangle \leq M\left\|B e_{i}\right\|^{2},
$$

for $i \in I$, which, by summation shows that

$$
\frac{\operatorname{tr}\left(|B|^{2} A\right)}{\operatorname{tr}\left(|B|^{2}\right)} \in[m, M] .
$$

The inequality (2.4) implies in the operator order of $\mathcal{B}(H)$ that

$$
f(A)-f\left(\frac{\operatorname{tr}\left(|B|^{2} A\right)}{\operatorname{tr}\left(|B|^{2}\right)}\right) 1_{H} \geq \delta_{f}\left(\frac{\operatorname{tr}\left(|B|^{2} A\right)}{\operatorname{tr}\left(|B|^{2}\right)}\right)\left(A-\frac{\operatorname{tr}\left(|B|^{2} A\right)}{\operatorname{tr}\left(|B|^{2}\right)} 1_{H}\right)
$$

which can be written as

$$
\begin{gathered}
\langle f(A) y, y\rangle-f\left(\frac{\operatorname{tr}\left(|B|^{2} A\right)}{\operatorname{tr}\left(|B|^{2}\right)}\right)\langle y, y\rangle \\
\geq \delta_{f}\left(\frac{\operatorname{tr}\left(|B|^{2} A\right)}{\operatorname{tr}\left(|B|^{2}\right)}\right)\left(\langle A y, y\rangle-\frac{\operatorname{tr}\left(|B|^{2} A\right)}{\operatorname{tr}\left(|B|^{2}\right)}\langle y, y\rangle\right),
\end{gathered}
$$

for any $y \in H$. This inequality is also of interest in itself.

Taking in (2.6) $y=B e_{i}$ we get

$$
\begin{gathered}
\left\langle f(A) B e_{i}, B e_{i}\right\rangle-f\left(\frac{\operatorname{tr}\left(|B|^{2} A\right)}{\operatorname{tr}\left(|B|^{2}\right)}\right)\left\langle B e_{i}, B e_{i}\right\rangle \\
\geq \delta_{f}\left(\frac{\operatorname{tr}\left(|B|^{2} A\right)}{\operatorname{tr}\left(|B|^{2}\right)}\right)\left(\left\langle A B e_{i}, B e_{i}\right\rangle-\frac{\operatorname{tr}\left(|B|^{2} A\right)}{\operatorname{tr}\left(|B|^{2}\right)}\left\langle B e_{i}, B e_{i}\right\rangle\right),
\end{gathered}
$$

which is equivalent to

$$
\begin{gathered}
\left\langle B^{*} f(A) B e_{i}, e_{i}\right\rangle-f\left(\frac{\operatorname{tr}\left(|B|^{2} A\right)}{\operatorname{tr}\left(|B|^{2}\right)}\right)\left\langle|B|^{2} e_{i}, e_{i}\right\rangle \\
\geq \delta_{f}\left(\frac{\operatorname{tr}\left(|B|^{2} A\right)}{\operatorname{tr}\left(|B|^{2}\right)}\right)\left(\left\langle B^{*} A B e_{i}, e_{i}\right\rangle-\frac{\operatorname{tr}\left(|B|^{2} A\right)}{\operatorname{tr}\left(|B|^{2}\right)}\left\langle|B|^{2} e_{i}, e_{i}\right\rangle\right),
\end{gathered}
$$

for any $i \in I$. 
Summing in (2.7) we get

$$
\begin{gathered}
\sum_{i \in I}\left\langle B^{*} f(A) B e_{i}, e_{i}\right\rangle-f\left(\frac{\operatorname{tr}\left(|B|^{2} A\right)}{\operatorname{tr}\left(|B|^{2}\right)}\right) \sum_{i \in I}\left\langle|B|^{2} e_{i}, e_{i}\right\rangle \\
\geq \delta_{f}\left(\frac{\operatorname{tr}\left(|B|^{2} A\right)}{\operatorname{tr}\left(|B|^{2}\right)}\right)\left(\sum_{i \in I}\left\langle B^{*} A B e_{i}, e_{i}\right\rangle-\frac{\operatorname{tr}\left(|B|^{2} A\right)}{\operatorname{tr}\left(|B|^{2}\right)} \sum_{i \in I}\left\langle|B|^{2} e_{i}, e_{i}\right\rangle\right) .
\end{gathered}
$$

However

$$
\begin{gathered}
\sum_{i \in I}\left\langle B^{*} f(A) B e_{i}, e_{i}\right\rangle=\sum_{i \in I}\left\langle B B^{*} f(A) e_{i}, e_{i}\right\rangle \\
=\sum_{i \in I}\left\langle\left|B^{*}\right|^{2} f(A) e_{i}, e_{i}\right\rangle=\operatorname{tr}\left(\left|B^{*}\right|^{2} f(A)\right)
\end{gathered}
$$

and

$$
\sum_{i \in I}\left\langle B^{*} A B e_{i}, e_{i}\right\rangle=\sum_{i \in I}\left\langle B B^{*} A e_{i}, e_{i}\right\rangle=\operatorname{tr}\left(\left|B^{*}\right|^{2} A\right) .
$$

By (2.8) we get

$$
\begin{gathered}
\operatorname{tr}\left(\left|B^{*}\right|^{2} f(A)\right)-f\left(\frac{\operatorname{tr}\left(|B|^{2} A\right)}{\operatorname{tr}\left(|B|^{2}\right)}\right) \operatorname{tr}\left(|B|^{2}\right) \\
\geq \delta_{f}\left(\frac{\operatorname{tr}\left(|B|^{2} A\right)}{\operatorname{tr}\left(|B|^{2}\right)}\right)\left(\operatorname{tr}\left(\left|B^{*}\right|^{2} A\right)-\operatorname{tr}\left(|B|^{2} A\right)\right),
\end{gathered}
$$

and the inequality (2.2) is thus proved.

Taking in (2.6) $y=B^{*} e_{i}$ we also get

$$
\begin{gathered}
\left\langle f(A) B^{*} e_{i}, B^{*} e_{i}\right\rangle-f\left(\frac{\operatorname{tr}\left(|B|^{2} A\right)}{\operatorname{tr}\left(|B|^{2}\right)}\right)\left\langle B^{*} e_{i}, B^{*} e_{i}\right\rangle \\
\geq \delta_{f}\left(\frac{\operatorname{tr}\left(|B|^{2} A\right)}{\operatorname{tr}\left(|B|^{2}\right)}\right)\left(\left\langle A B^{*} e_{i}, B^{*} e_{i}\right\rangle-\frac{\operatorname{tr}\left(|B|^{2} A\right)}{\operatorname{tr}\left(|B|^{2}\right)}\left\langle B^{*} e_{i}, B^{*} e_{i}\right\rangle\right),
\end{gathered}
$$

which is equivalent to

$$
\begin{gathered}
\left\langle B f(A) B^{*} e_{i}, e_{i}\right\rangle-f\left(\frac{\operatorname{tr}\left(|B|^{2} A\right)}{\operatorname{tr}\left(|B|^{2}\right)}\right)\left\langle B B^{*} e_{i}, e_{i}\right\rangle \\
\geq \delta_{f}\left(\frac{\operatorname{tr}\left(|B|^{2} A\right)}{\operatorname{tr}\left(|B|^{2}\right)}\right)\left(\left\langle B A B^{*} e_{i}, e_{i}\right\rangle-\frac{\operatorname{tr}\left(|B|^{2} A\right)}{\operatorname{tr}\left(|B|^{2}\right)}\left\langle B B^{*} e_{i}, e_{i}\right\rangle\right),
\end{gathered}
$$

for any $i \in I$.

Summing in (2.10) we get

$$
\begin{gathered}
\sum_{i \in I}\left\langle B f(A) B^{*} e_{i}, e_{i}\right\rangle-f\left(\frac{\operatorname{tr}\left(|B|^{2} A\right)}{\operatorname{tr}\left(|B|^{2}\right)}\right) \sum_{i \in I}\left\langle B B^{*} e_{i}, e_{i}\right\rangle \\
\geq \delta_{f}\left(\frac{\operatorname{tr}\left(|B|^{2} A\right)}{\operatorname{tr}\left(|B|^{2}\right)}\right)\left(\sum_{i \in I}\left\langle B A B^{*} e_{i}, e_{i}\right\rangle-\frac{\operatorname{tr}\left(|B|^{2} A\right)}{\operatorname{tr}\left(|B|^{2}\right)} \sum_{i \in I}\left\langle B B^{*} e_{i}, e_{i}\right\rangle\right) .
\end{gathered}
$$


Since

$$
\begin{gathered}
\sum_{i \in I}\left\langle B f(A) B^{*} e_{i}, e_{i}\right\rangle=\operatorname{tr}\left(B f(A) B^{*}\right)=\operatorname{tr}\left(B^{*} B f(A)\right)=\operatorname{tr}\left(|B|^{2} f(A)\right) \\
\sum_{i \in I}\left\langle B B^{*} e_{i}, e_{i}\right\rangle=\operatorname{tr}\left(B B^{*}\right)=\operatorname{tr}\left(B^{*} B\right)=\operatorname{tr}\left(|B|^{2}\right)
\end{gathered}
$$

and

$$
\sum_{i \in I}\left\langle B A B^{*} e_{i}, e_{i}\right\rangle=\operatorname{tr}\left(B A B^{*}\right)=\operatorname{tr}\left(B^{*} B A\right)=\operatorname{tr}\left(|B|^{2} A\right),
$$

then by (2.11) we get

$$
\operatorname{tr}\left(|B|^{2} f(A)\right)-f\left(\frac{\operatorname{tr}\left(|B|^{2} A\right)}{\operatorname{tr}\left(|B|^{2}\right)}\right) \operatorname{tr}\left(|B|^{2}\right) \geq 0
$$

and the inequality (2.3) is obtained.

Remark 2.1. The inequality (2.3) is obviously not as good as the first part of (1.12). However it is the natural alternative of Jensen's inequality for trace and provides simple and nice examples for various convex functions of interest. The proof here is also simpler than the one from [26] and has some natural reverses as follows.

Corollary 2.1. Let $A$ be a selfadjoint operator on the Hilbert space $H$ and assume that $\operatorname{Sp}(A) \subseteq[m, M]$ for some scalars $m, M$ with $m<M$. If $f$ is a continuous convex function on $[m, M]$ and $P \in \mathcal{B}_{1}(H) \backslash\{0\}, P \geq 0$ then $\frac{\operatorname{tr}(P A)}{\operatorname{tr}(P)} \in[m, M]$ and

$$
f\left(\frac{\operatorname{tr}(P A)}{\operatorname{tr}(P)}\right) \leq \frac{\operatorname{tr}(P f(A))}{\operatorname{tr}(P)} .
$$

The proof follows by either (2.2) or (2.3) on choosing $B=P^{1 / 2}, P \in \mathcal{B}_{1}(H) \backslash\{0\}$, $P \geq 0$.

The following lemma is of interest in itself:

Lemma 2.1. Let $S$ be a selfadjoint operator such that $\gamma 1_{H} \leq S \leq \Gamma 1_{H}$ for some real constants $\Gamma \geq \gamma$. Then for any $B \in \mathcal{B}_{2}(H) \backslash\{0\}$ we have

$$
\begin{gathered}
0 \leq \frac{\operatorname{tr}\left(|B|^{2} S^{2}\right)}{\operatorname{tr}\left(|B|^{2}\right)}-\left(\frac{\operatorname{tr}\left(|B|^{2} S\right)}{\operatorname{tr}\left(|B|^{2}\right)}\right)^{2} \\
\leq \frac{1}{2}(\Gamma-\gamma) \frac{1}{\operatorname{tr}\left(|B|^{2}\right)} \operatorname{tr}\left(|B|^{2}\left|S-\frac{\operatorname{tr}\left(|B|^{2} S\right)}{\operatorname{tr}\left(|B|^{2}\right)} 1_{H}\right|\right) \\
\leq \frac{1}{2}(\Gamma-\gamma)\left[\frac{\operatorname{tr}\left(|B|^{2} S^{2}\right)}{\operatorname{tr}\left(|B|^{2}\right)}-\left(\frac{\operatorname{tr}\left(|B|^{2} S\right)}{\operatorname{tr}\left(|B|^{2}\right)}\right)^{2}\right]^{1 / 2} \leq \frac{1}{4}(\Gamma-\gamma)^{2} .
\end{gathered}
$$


Proof. The first inequality follows by Jensen's inequality (2.3) for the convex function $f(t)=t^{2}$.

Now, observe that

$$
\begin{gathered}
\frac{1}{\operatorname{tr}\left(|B|^{2}\right)} \operatorname{tr}\left(|B|^{2}\left(S-\frac{\Gamma+\gamma}{2} 1_{H}\right)\left(S-\frac{\operatorname{tr}\left(|B|^{2} S\right)}{\operatorname{tr}\left(|B|^{2}\right)} 1_{H}\right)\right) \\
=\frac{1}{\operatorname{tr}\left(|B|^{2}\right)} \operatorname{tr}\left(|B|^{2} S\left(S-\frac{\operatorname{tr}\left(|B|^{2} S\right)}{\operatorname{tr}\left(|B|^{2}\right)} 1_{H}\right)\right) \\
-\frac{\Gamma+\gamma}{2} \frac{1}{\operatorname{tr}\left(|B|^{2}\right)} \operatorname{tr}\left(|B|^{2}\left(S-\frac{\operatorname{tr}\left(|B|^{2} S\right)}{\operatorname{tr}\left(|B|^{2}\right)} 1_{H}\right)\right) \\
=\frac{\operatorname{tr}\left(|B|^{2} S^{2}\right)}{\operatorname{tr}\left(|B|^{2}\right)}-\left(\frac{\operatorname{tr}\left(|B|^{2} S\right)}{\operatorname{tr}\left(|B|^{2}\right)}\right)^{2}
\end{gathered}
$$

since, obviously

$$
\operatorname{tr}\left(|B|^{2}\left(S-\frac{\operatorname{tr}\left(|B|^{2} S\right)}{\operatorname{tr}\left(|B|^{2}\right)} 1_{H}\right)\right)=0 .
$$

Now, since $\gamma 1_{H} \leq S \leq \Gamma 1_{H}$ then

$$
\left|S-\frac{\Gamma+\gamma}{2} 1_{H}\right| \leq \frac{1}{2}(\Gamma-\gamma)
$$

Taking the modulus in (2.14) and using the properties of trace, we have

$$
\begin{gathered}
\frac{\operatorname{tr}\left(|B|^{2} S^{2}\right)}{\operatorname{tr}\left(|B|^{2}\right)}-\left(\frac{\operatorname{tr}\left(|B|^{2} S\right)}{\operatorname{tr}\left(|B|^{2}\right)}\right)^{2} \\
=\frac{1}{\operatorname{tr}\left(|B|^{2}\right)}\left|\operatorname{tr}\left(|B|^{2}\left(S-\frac{\Gamma+\gamma}{2} 1_{H}\right)\left(S-\frac{\operatorname{tr}\left(|B|^{2} S\right)}{\operatorname{tr}\left(|B|^{2}\right)} 1_{H}\right)\right)\right| \\
\leq \frac{1}{\operatorname{tr}\left(|B|^{2}\right)} \operatorname{tr}\left(|B|^{2}\left|\left(S-\frac{\Gamma+\gamma}{2} 1_{H}\right)\left(S-\frac{\operatorname{tr}\left(|B|^{2} S\right)}{\operatorname{tr}\left(|B|^{2}\right)} 1_{H}\right)\right|\right) \\
\leq \frac{1}{2}(\Gamma-\gamma) \frac{1}{\operatorname{tr}\left(|B|^{2}\right)} \operatorname{tr}\left(|B|^{2}\left|S-\frac{\operatorname{tr}\left(|B|^{2} S\right)}{\operatorname{tr}\left(|B|^{2}\right)} 1_{H}\right|\right),
\end{gathered}
$$

which proves the first part of (2.13).

By Schwarz inequality for trace we also have

$$
\begin{gathered}
\frac{1}{\operatorname{tr}\left(|B|^{2}\right)} \operatorname{tr}\left(|B|^{2}\left|S-\frac{\operatorname{tr}\left(|B|^{2} S\right)}{\operatorname{tr}\left(|B|^{2}\right)} 1_{H}\right|\right) \\
\leq\left[\frac{1}{\operatorname{tr}\left(|B|^{2}\right)} \operatorname{tr}\left(|B|^{2}\left(S-\frac{\operatorname{tr}\left(|B|^{2} S\right)}{\operatorname{tr}\left(|B|^{2}\right)} 1_{H}\right)^{2}\right)\right]^{1 / 2} \\
=\left[\frac{\operatorname{tr}\left(|B|^{2} S^{2}\right)}{\operatorname{tr}\left(|B|^{2}\right)}-\left(\frac{\operatorname{tr}\left(|B|^{2} S\right)}{\operatorname{tr}\left(|B|^{2}\right)}\right)^{2}\right]^{1 / 2} .
\end{gathered}
$$


From (2.15) and (2.16) we get

$$
\begin{gathered}
\frac{\operatorname{tr}\left(|B|^{2} S^{2}\right)}{\operatorname{tr}\left(|B|^{2}\right)}-\left(\frac{\operatorname{tr}\left(|B|^{2} S\right)}{\operatorname{tr}\left(|B|^{2}\right)}\right)^{2} \\
\leq \frac{1}{2}(\Gamma-\gamma)\left[\frac{\operatorname{tr}\left(|B|^{2} S^{2}\right)}{\operatorname{tr}\left(|B|^{2}\right)}-\left(\frac{\operatorname{tr}\left(|B|^{2} S\right)}{\operatorname{tr}\left(|B|^{2}\right)}\right)^{2}\right]^{1 / 2},
\end{gathered}
$$

which implies that

$$
\left[\frac{\operatorname{tr}\left(|B|^{2} S^{2}\right)}{\operatorname{tr}\left(|B|^{2}\right)}-\left(\frac{\operatorname{tr}\left(|B|^{2} S\right)}{\operatorname{tr}\left(|B|^{2}\right)}\right)^{2}\right]^{1 / 2} \leq \frac{1}{2}(\Gamma-\gamma) .
$$

By (2.16) we then obtain

$$
\begin{gathered}
\frac{1}{\operatorname{tr}\left(|B|^{2}\right)} \operatorname{tr}\left(|B|^{2}\left|S-\frac{\operatorname{tr}\left(|B|^{2} S\right)}{\operatorname{tr}\left(|B|^{2}\right)} 1_{H}\right|\right) \\
\leq\left[\frac{\operatorname{tr}\left(|B|^{2} S^{2}\right)}{\operatorname{tr}\left(|B|^{2}\right)}-\left(\frac{\operatorname{tr}\left(|B|^{2} S\right)}{\operatorname{tr}\left(|B|^{2}\right)}\right)^{2}\right]^{1 / 2} \leq \frac{1}{2}(\Gamma-\gamma)
\end{gathered}
$$

that proves the last part of (2.13).

Remark 2.2. Let $S$ be a selfadjoint operator such that $\gamma 1_{H} \leq S \leq \Gamma 1_{H}$ for some real constants $\Gamma \geq \gamma$. Then for any $P \in \mathcal{B}_{1}(H) \backslash\{0\}, P \geq 0$ we have

$$
\begin{gathered}
0 \leq \frac{\operatorname{tr}\left(P S^{2}\right)}{\operatorname{tr}(P)}-\left(\frac{\operatorname{tr}(P S)}{\operatorname{tr}(P)}\right)^{2} \\
\leq \frac{1}{2}(\Gamma-\gamma) \frac{1}{\operatorname{tr}(P)} \operatorname{tr}\left(P\left|S-\frac{\operatorname{tr}(P S)}{\operatorname{tr}(P)} 1_{H}\right|\right) \\
\leq \frac{1}{2}(\Gamma-\gamma)\left[\frac{\operatorname{tr}\left(P S^{2}\right)}{\operatorname{tr}(P)}-\left(\frac{\operatorname{tr}(P S)}{\operatorname{tr}(P)}\right)^{2}\right]^{1 / 2} \leq \frac{1}{4}(\Gamma-\gamma)^{2} .
\end{gathered}
$$

The following result provides reverses for the inequalities (2.2) and (2.3) above:

Theorem 2.2. Let $A$ be a selfadjoint operator on the Hilbert space $H$ and assume that $S p(A) \subseteq[m, M]$ for some scalars $m, M$ with $m<M$. If $f$ is a continuously differentiable convex function on $[m, M]$ and $B \in \mathcal{B}_{2}(H) \backslash\{0\}$, then we have

$$
\begin{gathered}
0 \leq \frac{\operatorname{tr}\left(\left|B^{*}\right|^{2} f(A)\right)}{\operatorname{tr}\left(|B|^{2}\right)}-f\left(\frac{\operatorname{tr}\left(|B|^{2} A\right)}{\operatorname{tr}\left(|B|^{2}\right)}\right) \\
\leq \frac{\operatorname{tr}\left(\left|B^{*}\right|^{2} f^{\prime}(A) A\right)}{\operatorname{tr}\left(|B|^{2}\right)}-\frac{\operatorname{tr}\left(|B|^{2} A\right)}{\operatorname{tr}\left(|B|^{2}\right)} \cdot \frac{\operatorname{tr}\left(\left|B^{*}\right|^{2} f^{\prime}(A)\right)}{\operatorname{tr}\left(|B|^{2}\right)}
\end{gathered}
$$

and

$$
\begin{gathered}
0 \leq \frac{\operatorname{tr}\left(|B|^{2} f(A)\right)}{\operatorname{tr}\left(|B|^{2}\right)}-f\left(\frac{\operatorname{tr}\left(|B|^{2} A\right)}{\operatorname{tr}\left(|B|^{2}\right)}\right) \\
\leq \frac{\operatorname{tr}\left(|B|^{2} f^{\prime}(A) A\right)}{\operatorname{tr}\left(|B|^{2}\right)}-\frac{\operatorname{tr}\left(|B|^{2} A\right)}{\operatorname{tr}\left(|B|^{2}\right)} \cdot \frac{\operatorname{tr}\left(|B|^{2} f^{\prime}(A)\right)}{\operatorname{tr}\left(|B|^{2}\right)}=: \mathcal{K}\left(f^{\prime}, B, A\right) .
\end{gathered}
$$


Moreover, we have

$$
\begin{aligned}
& \mathcal{K}\left(f^{\prime}, B, A\right) \\
& \leq\left\{\begin{array}{l}
\frac{1}{2}\left[f^{\prime}(M)-f^{\prime}(m)\right] \frac{\operatorname{tr}\left(|B|^{2}\left|A-\frac{\operatorname{tr}\left(|B|^{2} A\right)}{\operatorname{tr}\left(|B|^{2}\right)} 1_{H}\right|\right)}{\operatorname{tr}\left(|B|^{2}\right)} \\
\frac{1}{2}(M-m) \frac{\operatorname{tr}\left(|B|^{2}\left|f^{\prime}(A)-\frac{\operatorname{tr}\left(|B|^{2} f^{\prime}(A)\right)}{\operatorname{tr}\left(|B|^{2}\right)} 1_{H}\right|\right)}{\operatorname{tr}\left(|B|^{2}\right)}
\end{array}\right. \\
& \leq\left\{\begin{array}{l}
\frac{1}{2}\left[f^{\prime}(M)-f^{\prime}(m)\right]\left[\frac{\operatorname{tr}\left(|B|^{2} A^{2}\right)}{\operatorname{tr}\left(|B|^{2}\right)}-\left(\frac{\operatorname{tr}\left(|B|^{2} A\right)}{\operatorname{tr}\left(|B|^{2}\right)}\right)^{2}\right]^{1 / 2} \\
\frac{1}{2}(M-m)\left[\frac{\operatorname{tr}\left(|B|^{2}\left[f^{\prime}(A)\right]^{2}\right)}{\operatorname{tr}\left(|B|^{2}\right)}-\left(\frac{\operatorname{tr}\left(|B|^{2} f^{\prime}(A)\right)}{\operatorname{tr}\left(|B|^{2}\right)}\right)^{2}\right]^{1 / 2}
\end{array}\right. \\
& \leq \frac{1}{4}\left[f^{\prime}(M)-f^{\prime}(m)\right](M-m) \text {. }
\end{aligned}
$$

Proof. By the gradient inequality we have

$$
f(\tau)-f(\varsigma) \leq f^{\prime}(\tau)(\tau-\varsigma)
$$

for any $\tau, \varsigma \in[m, M]$.

This inequality implies in the operator order

$$
f(A)-f\left(\frac{\operatorname{tr}\left(|B|^{2} A\right)}{\operatorname{tr}\left(|B|^{2}\right)}\right) 1_{H} \leq f^{\prime}(A)\left(A-\frac{\operatorname{tr}\left(|B|^{2} A\right)}{\operatorname{tr}\left(|B|^{2}\right)} 1_{H}\right)
$$

that is equivalent to

$$
\begin{gathered}
\langle f(A) y, y\rangle-f\left(\frac{\operatorname{tr}\left(|B|^{2} A\right)}{\operatorname{tr}\left(|B|^{2}\right)}\right)\langle y, y\rangle \\
\leq\left\langle f^{\prime}(A) A y, y\right\rangle-\frac{\operatorname{tr}\left(|B|^{2} A\right)}{\operatorname{tr}\left(|B|^{2}\right)}\left\langle f^{\prime}(A) y, y\right\rangle
\end{gathered}
$$

for any $y \in H$, which is of interest in itself as well.

Let $\mathcal{E}:=\left\{e_{i}\right\}_{i \in I}$ be an orthonormal basis in $H$.

If we take in (2.22) $y=B e_{i}$ and sum, then we get

$$
\begin{gathered}
\sum_{i \in I}\left\langle f(A) B e_{i}, B e_{i}\right\rangle-f\left(\frac{\operatorname{tr}\left(|B|^{2} A\right)}{\operatorname{tr}\left(|B|^{2}\right)}\right) \sum_{i \in I}\left\langle B e_{i}, B e_{i}\right\rangle \\
\leq \sum_{i \in I}\left\langle f^{\prime}(A) A B e_{i}, B e_{i}\right\rangle-\frac{\operatorname{tr}\left(|B|^{2} A\right)}{\operatorname{tr}\left(|B|^{2}\right)} \sum_{i \in I}\left\langle f^{\prime}(A) B e_{i}, B e_{i}\right\rangle,
\end{gathered}
$$


which is equivalent to

$$
\begin{aligned}
& \sum_{i \in I}\left\langle B^{*} f(A) B e_{i}, e_{i}\right\rangle-f\left(\frac{\operatorname{tr}\left(|B|^{2} A\right)}{\operatorname{tr}\left(|B|^{2}\right)}\right) \sum_{i \in I}\left\langle B^{*} B e_{i}, e_{i}\right\rangle \\
\leq & \sum_{i \in I}\left\langle B^{*} f^{\prime}(A) A B e_{i}, e_{i}\right\rangle-\frac{\operatorname{tr}\left(|B|^{2} A\right)}{\operatorname{tr}\left(|B|^{2}\right)} \sum_{i \in I}\left\langle B^{*} f^{\prime}(A) B e_{i}, e_{i}\right\rangle
\end{aligned}
$$

and the inequality (2.18) is obtained.

If we take in (2.22) $y=B^{*} e_{i}$ and sum, then we get

$$
\begin{aligned}
& \sum_{i \in I}\left\langle f(A) B^{*} e_{i}, B^{*} e_{i}\right\rangle-f\left(\frac{\operatorname{tr}\left(|B|^{2} A\right)}{\operatorname{tr}\left(|B|^{2}\right)}\right) \sum_{i \in I}\left\langle B^{*} e_{i}, B^{*} e_{i}\right\rangle \\
\leq & \sum_{i \in I}\left\langle f^{\prime}(A) A B^{*} e_{i}, B^{*} e_{i}\right\rangle-\frac{\operatorname{tr}\left(|B|^{2} A\right)}{\operatorname{tr}\left(|B|^{2}\right)} \sum_{i \in I}\left\langle f^{\prime}(A) B^{*} e_{i}, B^{*} e_{i}\right\rangle
\end{aligned}
$$

that is equivalent to

$$
\begin{gathered}
\sum_{i \in I}\left\langle B f(A) B^{*} e_{i}, e_{i}\right\rangle-f\left(\frac{\operatorname{tr}\left(|B|^{2} A\right)}{\operatorname{tr}\left(|B|^{2}\right)}\right) \sum_{i \in I}\left\langle B B^{*} e_{i}, e_{i}\right\rangle \\
\leq \sum_{i \in I}\left\langle B f^{\prime}(A) A B^{*} e_{i}, e_{i}\right\rangle-\frac{\operatorname{tr}\left(|B|^{2} A\right)}{\operatorname{tr}\left(|B|^{2}\right)} \sum_{i \in I}\left\langle B f^{\prime}(A) B^{*} e_{i}, e_{i}\right\rangle
\end{gathered}
$$

and the inequality (2.19) is obtained.

Now, since $f$ is continuously convex on $[m, M]$, then $f^{\prime}$ is monotonic nondecreasing on $[m, M]$ and $f^{\prime}(m) \leq f^{\prime}(t) \leq f^{\prime}(M)$ for any $t \in[m, M]$. We also observe that

$$
\begin{gathered}
\frac{1}{\operatorname{tr}\left(|B|^{2}\right)} \operatorname{tr}\left(|B|^{2}\left[f^{\prime}(A)-\frac{f^{\prime}(m)+f^{\prime}(M)}{2} 1_{H}\right]\left[A-\frac{\operatorname{tr}\left(|B|^{2} A\right)}{\operatorname{tr}\left(|B|^{2}\right)} 1_{H}\right]\right) \\
=\frac{1}{\operatorname{tr}\left(|B|^{2}\right)} \operatorname{tr}\left(|B|^{2} f^{\prime}(A)\left[A-\frac{\operatorname{tr}\left(|B|^{2} A\right)}{\operatorname{tr}\left(|B|^{2}\right)} 1_{H}\right]\right) \\
-\frac{f^{\prime}(m)+f^{\prime}(M)}{2} \frac{1}{\operatorname{tr}\left(|B|^{2}\right)} \operatorname{tr}\left(|B|^{2}\left[A-\frac{\operatorname{tr}\left(|B|^{2} A\right)}{\operatorname{tr}\left(|B|^{2}\right)} 1_{H}\right]\right) \\
=\mathcal{K}\left(f^{\prime}, B, A\right) .
\end{gathered}
$$

Since

$$
\left|f^{\prime}(A)-\frac{f^{\prime}(m)+f^{\prime}(M)}{2} 1_{H}\right| \leq \frac{1}{2}\left[f^{\prime}(M)-f^{\prime}(m)\right] 1_{H},
$$

then by taking the modulus in (2.24) and utilizing the properties of trace we have

$$
\begin{gathered}
0 \leq \mathcal{K}\left(f^{\prime}, B, A\right) \\
\leq \frac{1}{\operatorname{tr}\left(|B|^{2}\right)} \\
\times \operatorname{tr}\left(|B|^{2}\left|\left[f^{\prime}(A)-\frac{f^{\prime}(m)+f^{\prime}(M)}{2} 1_{H}\right]\left[A-\frac{\operatorname{tr}\left(|B|^{2} A\right)}{\operatorname{tr}\left(|B|^{2}\right)} 1_{H}\right]\right|\right) \\
\leq \frac{1}{2}\left[f^{\prime}(M)-f^{\prime}(m)\right] \frac{1}{\operatorname{tr}\left(|B|^{2}\right)} \operatorname{tr}\left(|B|^{2}\left|A-\frac{\operatorname{tr}\left(|B|^{2} A\right)}{\operatorname{tr}\left(|B|^{2}\right)} 1_{H}\right|\right),
\end{gathered}
$$


and the first inequality in the first branch of (2.20) is proved.

We have $m 1_{H} \leq A \leq M 1_{H}$ and by applying Lemma 2.1 we can state that

$$
\begin{gathered}
\frac{1}{\operatorname{tr}\left(|B|^{2}\right)} \operatorname{tr}\left(|B|^{2}\left|A-\frac{\operatorname{tr}\left(|B|^{2} A\right)}{\operatorname{tr}\left(|B|^{2}\right)} 1_{H}\right|\right) \\
\leq\left[\frac{\operatorname{tr}\left(|B|^{2} A^{2}\right)}{\operatorname{tr}\left(|B|^{2}\right)}-\left(\frac{\operatorname{tr}\left(|B|^{2} A\right)}{\operatorname{tr}\left(|B|^{2}\right)}\right)^{2}\right]^{1 / 2} \leq \frac{1}{2}(M-m) .
\end{gathered}
$$

Making use of (2.25) and (2.26) we deduce the second and the third inequalities in the first branch of $(2.20)$.

We observe that $\mathcal{K}\left(f^{\prime}, B, A\right)$ can be also represented as

$$
\begin{gathered}
\mathcal{K}\left(f^{\prime}, B, A\right) \\
=\frac{1}{\operatorname{tr}\left(|B|^{2}\right)} \operatorname{tr}\left(|B|^{2}\left[f^{\prime}(A)-\frac{\operatorname{tr}\left(|B|^{2} f^{\prime}(A)\right)}{\operatorname{tr}\left(|B|^{2}\right)} 1_{H}\right]\left(A-\frac{m+M}{2} 1_{H}\right)\right) .
\end{gathered}
$$

Applying a similar argument as above for this representation, we get the second branch of the inequality (2.20).

The proof is complete.

Corollary 2.2. Let $A$ be a selfadjoint operator on the Hilbert space $H$ and assume that $\operatorname{Sp}(A) \subseteq[m, M]$ for some scalars $m, M$ with $m<M$. If $f$ is a continuously differentiable convex function on $[m, M]$ and $P \in \mathcal{B}_{1}(H) \backslash\{0\}, P \geq 0$, then we have

$$
\begin{aligned}
& 0 \leq \frac{\operatorname{tr}(P f(A))}{\operatorname{tr}(P)}-f\left(\frac{\operatorname{tr}(P A)}{\operatorname{tr}(P)}\right) \\
& \leq \frac{\operatorname{tr}\left(P f^{\prime}(A) A\right)}{\operatorname{tr}(P)}-\frac{\operatorname{tr}(P A)}{\operatorname{tr}(P)} \cdot \frac{\operatorname{tr}\left(P f^{\prime}(A)\right)}{\operatorname{tr}(P)} \\
& \leq\left\{\begin{array}{l}
\frac{1}{2}\left[f^{\prime}(M)-f^{\prime}(m)\right] \frac{\operatorname{tr}\left(P\left|A-\frac{\operatorname{tr}(P A)}{\operatorname{tr}(P)} 1_{H}\right|\right)}{\operatorname{tr}(P)} \\
\frac{1}{2}(M-m) \frac{\operatorname{tr}\left(P\left|f^{\prime}(A)-\frac{\operatorname{tr}\left(P f^{\prime}(A)\right)}{\operatorname{tr}(P)} 1_{H}\right|\right)}{\operatorname{tr}(P)}
\end{array}\right. \\
& \leq\left\{\begin{array}{l}
\frac{1}{2}\left[f^{\prime}(M)-f^{\prime}(m)\right]\left[\frac{\operatorname{tr}\left(P A^{2}\right)}{\operatorname{tr}(P)}-\left(\frac{\operatorname{tr}(P A)}{\operatorname{tr}(P)}\right)^{2}\right]^{1 / 2} \\
\frac{1}{2}(M-m)\left[\frac{\operatorname{tr}\left(P\left[f^{\prime}(A)\right]^{2}\right)}{\operatorname{tr}(P)}-\left(\frac{\operatorname{tr}\left(P f^{\prime}(A)\right)}{\operatorname{tr}(P)}\right)^{2}\right]^{1 / 2}
\end{array}\right. \\
& \leq \frac{1}{4}\left[f^{\prime}(M)-f^{\prime}(m)\right](M-m) \text {. }
\end{aligned}
$$

Remark 2.3. Let $\mathcal{M}_{n}(\mathbb{C})$ be the space of all square matrices of order $n$ with complex elements and $A \in \mathcal{M}_{n}(\mathbb{C})$ be a Hermitian matrix such that $S p(A) \subseteq[m, M]$ for some 
scalars $m, M$ with $m<M$. If $f$ is a continuously differentiable convex function on $[m, M]$, then by taking $P=I_{n}$, the identity matrix, in $(2.27)$ we get

$$
\begin{aligned}
& 0 \leq \frac{\operatorname{tr}(f(A))}{n}-f\left(\frac{\operatorname{tr}(A)}{n}\right) \\
& \leq \frac{\operatorname{tr}\left(f^{\prime}(A) A\right)}{n}-\frac{\operatorname{tr}(A)}{n} \cdot \frac{\operatorname{tr}\left(f^{\prime}(A)\right)}{n} \\
& \leq\left\{\begin{array}{l}
\frac{1}{2}\left[f^{\prime}(M)-f^{\prime}(m)\right] \frac{\operatorname{tr}\left(\left|A-\frac{\operatorname{tr}(A)}{n} I_{n}\right|\right)}{n} \\
\frac{1}{2}(M-m) \frac{\operatorname{tr}\left(\left|f^{\prime}(A)-\frac{\operatorname{tr}\left(f^{\prime}(A)\right)}{n} I_{n}\right|\right)}{n}
\end{array}\right. \\
& \leq\left\{\begin{array}{l}
\frac{1}{2}\left[f^{\prime}(M)-f^{\prime}(m)\right]\left[\frac{\operatorname{tr}\left(A^{2}\right)}{n}-\left(\frac{\operatorname{tr}(A)}{n}\right)^{2}\right]^{1 / 2} \\
\frac{1}{2}(M-m)\left[\frac{\operatorname{tr}\left(\left[f^{\prime}(A)\right]^{2}\right)}{n}-\left(\frac{\operatorname{tr}\left(f^{\prime}(A)\right)}{n}\right)^{2}\right]^{1 / 2}
\end{array}\right. \\
& \leq \frac{1}{4}\left[f^{\prime}(M)-f^{\prime}(m)\right](M-m) .
\end{aligned}
$$

\section{Some Examples}

We consider the power function $f:(0, \infty) \rightarrow(0, \infty), f(t)=t^{r}$ with $t \in \mathbb{R} \backslash\{0\}$. For $r \in(-\infty, 0) \cup[1, \infty), f$ is convex while for $r \in(0,1), f$ is concave. Denote $\mathcal{B}_{1}^{+}(H):=\left\{P\right.$ with $P \in \mathcal{B}_{1}(H)$ and $\left.P \geq 0\right\}$.

Let $r \geq 1$ and $A$ be a selfadjoint operator on the Hilbert space $H$ and assume that $S p(\bar{A}) \subseteq[m, M]$ for some scalars $m, M$ with $0 \leq m<M$. If $P \in \mathcal{B}_{1}^{+}(H) \backslash\{0\}$, then

$$
\begin{aligned}
& 0 \leq \frac{\operatorname{tr}\left(P A^{r}\right)}{\operatorname{tr}(P)}-\left(\frac{\operatorname{tr}(P A)}{\operatorname{tr}(P)}\right)^{r} \\
& \leq r\left[\frac{\operatorname{tr}\left(P A^{r}\right)}{\operatorname{tr}(P)}-\frac{\operatorname{tr}(P A)}{\operatorname{tr}(P)} \cdot \frac{\operatorname{tr}\left(P A^{r-1}\right)}{\operatorname{tr}(P)}\right] \\
& \leq\left\{\begin{array}{l}
\frac{1}{2} r\left(M^{r-1}-m^{r-1}\right) \frac{\operatorname{tr}\left(P\left|A-\frac{\operatorname{tr}(P A)}{\operatorname{tr}(P)} 1_{H}\right|\right)}{\operatorname{tr}(P)} \\
\frac{1}{2} r(M-m) \frac{\operatorname{tr}\left(P\left|A^{r-1}-\frac{\operatorname{tr}\left(P A^{r-1}\right)}{\operatorname{tr}(P)} 1_{H}\right|\right)}{\operatorname{tr}(P)}
\end{array}\right. \\
& \leq\left\{\begin{array}{l}
\frac{1}{2} r\left(M^{r-1}-m^{r-1}\right)\left[\frac{\operatorname{tr}\left(P A^{2}\right)}{\operatorname{tr}(P)}-\left(\frac{\operatorname{tr}(P A)}{\operatorname{tr}(P)}\right)^{2}\right]^{1 / 2} \\
\frac{1}{2} r(M-m)\left[\frac{\operatorname{tr}\left(P A^{2(r-1)}\right)}{\operatorname{tr}(P)}-\left(\frac{\operatorname{tr}\left(P A^{r-1}\right)}{\operatorname{tr}(P)}\right)^{2}\right]^{1 / 2}
\end{array}\right. \\
& \leq \frac{1}{4} r\left(M^{r-1}-m^{r-1}\right)(M-m) \text {. }
\end{aligned}
$$


Consider the convex function $f:(0, \infty) \rightarrow(0, \infty), f(t)=-\ln t$ and let $A$ be a selfadjoint operator on the Hilbert space $H$ and assume that $S p(A) \subseteq[m, M]$ for some scalars $m, M$ with $0<m<M$. If $P \in \mathcal{B}_{1}^{+}(H) \backslash\{0\}$, then

$$
\begin{aligned}
& 0 \leq \ln \left(\frac{\operatorname{tr}(P A)}{\operatorname{tr}(P)}\right)-\frac{\operatorname{tr}(P \ln A)}{\operatorname{tr}(P)} \\
& \leq \frac{\operatorname{tr}(P A)}{\operatorname{tr}(P)} \cdot \frac{\operatorname{tr}\left(P A^{-1}\right)}{\operatorname{tr}(P)}-1 \\
& \leq\left\{\begin{array}{l}
\frac{M-m}{2 m M} \frac{\operatorname{tr}\left(P\left|A-\frac{\operatorname{tr}(P A)}{\operatorname{tr}(P)} 1_{H}\right|\right)}{\operatorname{tr}(P)} \\
\frac{1}{2}(M-m) \frac{\operatorname{tr}\left(P\left|A^{-1}-\frac{\operatorname{tr}\left(P A^{-1}\right)}{\operatorname{tr}(P)} 1_{H}\right|\right)}{\operatorname{tr}(P)}
\end{array}\right. \\
& \leq\left\{\begin{array}{l}
\frac{M-m}{2 m M}\left[\frac{\operatorname{tr}\left(P A^{2}\right)}{\operatorname{tr}(P)}-\left(\frac{\operatorname{tr}(P A)}{\operatorname{tr}(P)}\right)^{2}\right]^{1 / 2} \\
\frac{1}{2}(M-m)\left[\frac{\operatorname{tr}\left(P A^{-2}\right)}{\operatorname{tr}(P)}-\left(\frac{\operatorname{tr}\left(P A^{-1}\right)}{\operatorname{tr}(P)}\right)^{2}\right]^{1 / 2}
\end{array}\right. \\
& \leq \frac{(M-m)^{2}}{4 m M} \text {. }
\end{aligned}
$$

Consider the convex function $f(t)=t \ln t$ and let $A$ be a selfadjoint operator on the Hilbert space $H$ and assume that $S p(A) \subseteq[m, M]$ for some scalars $m, M$ with $0<m<M$. If $P \in \mathcal{B}_{1}^{+}(H) \backslash\{0\}$, then

$$
\begin{aligned}
& 0 \leq \frac{\operatorname{tr}(P A \ln A)}{\operatorname{tr}(P)}-\frac{\operatorname{tr}(P A)}{\operatorname{tr}(P)} \ln \left(\frac{\operatorname{tr}(P A)}{\operatorname{tr}(P)}\right) \\
& \leq \frac{\operatorname{tr}(P A \ln (e A))}{\operatorname{tr}(P)}-\frac{\operatorname{tr}(P A)}{\operatorname{tr}(P)} \cdot \frac{\operatorname{tr}(P \ln (e A))}{\operatorname{tr}(P)} \\
& \leq\left\{\begin{array}{l}
\frac{1}{2} \ln \left(\frac{M}{m}\right) \frac{\operatorname{tr}\left(P\left|A-\frac{\operatorname{tr}(P A)}{\operatorname{tr}(P)} 1_{H}\right|\right)}{\operatorname{tr}(P)} \\
\frac{1}{2}(M-m) \frac{\operatorname{tr}\left(P\left|\ln (e A)-\frac{\operatorname{tr}(P \ln (e A))}{\operatorname{tr}(P)} 1_{H}\right|\right)}{\operatorname{tr}(P)}
\end{array}\right. \\
& \leq\left\{\begin{array}{l}
\frac{1}{2} \ln \left(\frac{M}{m}\right)\left[\frac{\operatorname{tr}\left(P A^{2}\right)}{\operatorname{tr}(P)}-\left(\frac{\operatorname{tr}(P A)}{\operatorname{tr}(P)}\right)^{2}\right]^{1 / 2} \\
\frac{1}{2}(M-m)\left[\frac{\operatorname{tr}\left(P[\ln (e A)]^{2}\right)}{\operatorname{tr}(P)}-\left(\frac{\operatorname{tr}(P \ln (e A))}{\operatorname{tr}(P)}\right)^{2}\right]^{1 / 2}
\end{array}\right. \\
& \leq \frac{1}{4}(M-m) \ln \left(\frac{M}{m}\right) \text {. }
\end{aligned}
$$




\section{R E F E R E N C E S}

1. T. Ando: Matrix Young inequalities, Oper. Theory Adv. Appl. 75 (1995), 33-38.

2. R. Bellman: Some inequalities for positive definite matrices, in: E. F. Beckenbach (Ed.), General Inequalities 2, Proceedings of the 2nd International Conference on General Inequalities, Birkhäuser, Basel, 1980, pp. 89-90.

3. E. V. BelmegA, M. Jungers and S. LAsulce : A generalization of a trace inequality for positive definite matrices. Aust. J. Math. Anal. Appl. 7 (2010), no. 2, Art. 26, 5 pp.

4. E. A. CARLEn: Trace inequalities and quantum entropy: an introductory course, [Online http://www.mathphys.org/AZschool/material/AZ09-carlen.pdf] .

5. D. Chang: A matrix trace inequality for products of Hermitian matrices, J. Math. Anal. Appl. 237 (1999) 721-725.

6. L. Chen and C. Wong: Inequalities for singular values and traces, Linear Algebra Appl. 171 (1992), 109-120.

7. I. D. Coop: On matrix trace inequalities and related topics for products of Hermitian matrix, J. Math. Anal. Appl. 188 (1994) 999-1001.

8. S. S. Dragomir: A converse result for Jensen's discrete inequality via Gruss' inequality and applications in information theory. An. Univ. Oradea Fasc. Mat. 7 (1999/2000), 178-189.

9. S. S. Dragomir: On a reverse of Jessen's inequality for isotonic linear functionals, J. Ineq. Pure \& Appl. Math., 2(2001), No. 3, Article 36.

10. S. S. Dragomir: A Grüss type inequality for isotonic linear functionals and applications. Demonstratio Math. 36 (2003), no. 3, 551-562. Preprint, RGMIA Res. Rep. Coll. 5(2002), Suplement, Art. 12. [ONLINE:http://rgmia.org/v5(E).php].

11. S. S. Dragomir: Bounds for the normalized Jensen functional, Bull. Austral. Math. Soc. 74 (3) (2006), 471-476.

12. S. S. Dragomir: Bounds for the deviation of a function from the chord generated by its extremities. Bull. Aust. Math. Soc. 78 (2008), no. 2, 225-248.

13. S. S. Dragomir: Grüss' type inequalities for functions of selfadjoint operators in Hilbert spaces, Preprint, RGMIA Res. Rep. Coll. 11(e) (2008), Art. 11. [ONLINE: http://rgmia.org/v11(E).php].

14. S. S. Dragomir: Some inequalities for convex functions of selfadjoint operators in Hilbert spaces, Filomat 23 (2009), No. 3, 81-92. Preprint, RGMIA Res. Rep. Coll. 11(e) (2008), Art. 10.

15. S. S. Dragomir: Some Jensen's type inequalities for twice differentiable functions of selfadjoint operators in Hilbert spaces, Filomat 23 (2009), No. 3, 211-222. Preprint, RGMIA Res. Rep. Coll. 11(e) (2008), Art. 13.

16. S. S. Dragomir: Some new Grüss' type inequalities for functions of selfadjoint operators in Hilbert spaces, Sarajevo J. Math. 6(18), (2010), No. 1, 89-107. Preprint RGMIA Res. Rep. Coll. 11(e) (2008), Art. 12. [ONLINE: http://rgmia.org/v11(E).php].

17. S. S. Dragomir: New bounds for the Čebyšev functional of two functions of selfadjoint operators in Hilbert spaces, Filomat 24 (2010), No. 2, 27-39. 
18. S. S. Dragomir: Some Jensen's type inequalities for log-convex functions of selfadjoint operators in Hilbert spaces, Bull. Malays. Math. Sci. Soc. 34 (2011), No. 3. Preprint, RGMIA Res. Rep. Coll., 13 (2010), Sup. Art. 2.

19. S. S. Dragomir: Some reverses of the Jensen inequality for functions of selfadjoint operators in Hilbert spaces, J. Ineq. \& Appl. Vol. 2010, Article ID 496821. Preprint, RGMIA Res. Rep. Coll. 11(e) (2008), Art. 15. [ONLINE: http://rgmia.org/v11(E) .php].

20. S. S. Dragomir: Some Slater's type inequalities for convex functions of selfadjoint operators in Hilbert spaces, Rev. Un. Mat. Argentina, 52 (2011), No.1, 109-120. Preprint, RGMIA Res. Rep. Coll. 11(e) (2008), Art. 7.

21. S. S. Dragomir: Hermite-Hadamard's type inequalities for operator convex functions, Appl. Math. Comp. 218 (2011), 766-772. Preprint, RGMIA Res. Rep. Coll. 13(2010), No. 1, Art. 7.

22. S. S. DRAGOMIR: Hermite-Hadamard's type inequalities for convex functions of selfadjoint operators in Hilbert spaces, Linear Algebra Appl. 436 (2012), no. 5, 1503-1515. Preprint, RGMIA Res. Rep. Coll. 13(2010), No. 2, Art 1.

23. S. S. Dragomir: New Jensen's type inequalities for differentiable log-convex functions of selfadjoint operators in Hilbert spaces, Sarajevo J. Math. 19 (2011), No. 1, 67-80. Preprint, RGMIA Res. Rep. Coll. 13(2010), Sup. Art. 2.

24. S. S. Dragomir: Operator Inequalities of the Jensen, Čebyšev and Grüss Type. Springer Briefs in Mathematics. Springer, New York, 2012. xii+121 pp. ISBN: 978-1-4614-1520-6.

25. S. S. DragomiR: Operator Inequalities of Ostrowski and Trapezoidal Type. Springer Briefs in Mathematics. Springer, New York, 2012. $\mathrm{x}+112$ pp. ISBN: 978-1-4614-1778-1

26. S. S. Dragomir: Some trace inequalities for convex functions of selfadjoint operators in Hilbert spaces, Korean J. Math. 24 (2016), No. 2, pp. 273-296, Preprint, RGMIA Res. Rep. Coll. 17 (2014), Art 115.

27. S. S. Dragomir and N. M. IONESCU: Some converse of Jensen's inequality and applications. Rev. Anal. Numér. Théor. Approx. 23 (1994), no. 1, 71-78. MR:1325895 (96c:26012).

28. S. Furuichi and M. Lin: Refinements of the trace inequality of Belmega, Lasaulce and Debbah. Aust. J. Math. Anal. Appl. 7 (2010), no. 2, Art. 23, 4 pp.

29. T. Furuta, J. MićIĆ Hot, J. PeČArić and Y. SeO: Mond-Pečarić Method in Operator Inequalities. Inequalities for Bounded Selfadjoint Operators on a Hilbert Space, Element, Zagreb, 2005.

30. G. Helmberg: Introduction to Spectral Theory in Hilbert Space, John Wiley, New York, 1969.

31. H. D. LeE: On some matrix inequalities, Korean J. Math. 16 (2008), No. 4, pp. 565-571.

32. L. Liv: A trace class operator inequality, J. Math. Anal. Appl. 328 (2007) 14841486.

33. S. Manjegani: Hölder and Young inequalities for the trace of operators, Positivity 11 (2007), 239-250.

34. H. Neudecker: A matrix trace inequality, J. Math. Anal. Appl. 166 (1992) 302-303. 
35. K. Shebrawi and H. Albadawi: Operator norm inequalities of Minkowski type, J. Inequal. Pure Appl. Math. 9(1) (2008), 1-10, article 26.

36. K. Shebrawi and H. Albadawi: Trace inequalities for matrices, Bull. Aust. Math. Soc. 87 (2013), 139-148.

37. B. Simon: Trace Ideals and Their Applications, Cambridge University Press, Cambridge, 1979.

38. A. Matković, J. PeČarić and I. Perić: A variant of Jensen's inequality of Mercer's type for operators with applications. Linear Algebra Appl. 418 (2006), no. 2-3, 551-564.

39. C. A. MCCARthy: $c_{p}$, Israel J. Math., 5(1967), 249-271.

40. J. Mićić, Y. Seo, S.-E. TAKahasi and M. Tominaga: Inequalities of Furuta and Mond-Pečarić, Math. Ineq. Appl. 2 (1999), 83-111.

41. B. Mond and J. PeČARIĆ: Convex inequalities in Hilbert space, Houston J. Math. 19 (1993), 405-420.

42. B. Mond and J. PEČARIĆ: On some operator inequalities, Indian J. Math. 35 (1993), 221-232.

43. B. Mond and J. PEČARIĆ: Classical inequalities for matrix functions, Utilitas Math. 46 (1994), 155-166.

44. S. Simić: On a global upper bound for Jensen's inequality, J. Math. Anal. Appl. 343 (2008), 414-419.

45. Z. UluköK and R. Türkmen: On some matrix trace inequalities. J. Inequal. Appl. 2010, Art. ID 201486, 8 pp.

46. X. Yang: A matrix trace inequality, J. Math. Anal. Appl. 250 (2000) 372-374.

47. X. M. YANG, X. Q. YANG and K. L. TEO: A matrix trace inequality, J. Math. Anal. Appl. 263 (2001), 327-331.

48. Y. YAng: A matrix trace inequality, J. Math. Anal. Appl. 133 (1988) 573-574.

Sever Silvestru Dragomir

College of Engineering

Victoria University

Ballarat Road

8001 Melbourne, Australia

sever.dragomir@vu.edu .au 